\title{
Empirical Results for Wideband Multidimensional Spectrum Usage
}

\author{
Khalid A. Qaraqe*, Hasari Celebi*, Ali Gorcin ${ }^{\dagger}$, Amer El-Saigh ${ }^{\ddagger}$, Huseyin Arslan ${ }^{\dagger}$, Mohamed-slim Alouini* \\ *Dept. of Electrical and Computer Engineering, Texas A\&M University at Qatar, PO Box 23874, Education City, Doha, Qatar \\ Email: hasari.celebi,ali.gorcin,khalid.qaraqe,alouini@qatar.tamu.edu \\ ${ }^{\dagger}$ Department of Electrical Engineering, University of South Florida, 4202 E. Fowler Ave., ENB-118, Tampa, FL, 33620 \\ Email: arslan@eng.usf.edu \\ $\ddagger$ Networks R\&D, Qatar Telecom (Qtel) Q.S.C, PO Box 217, Doha, Qatar \\ Email: aelsaigh@qtel.com.qa
}

\begin{abstract}
Cognitive Radio (CR) systems with spectrum awareness feature is a promising approach to use spectrum effectively. However, accurate modeling of spectrum utilization is crucial for the development and performance evaluation of such systems. Hence, in this paper, a wideband multidimensional spectrum occupancy measurement campaign is conducted to study the evolution of RF spectrum over time, frequency, and space dimensions simultaneously. The measurements are performed over three consecutive days considering $700-3000 \mathrm{MHz}$ frequency band at four different locations concurrently. The measurement results show low utilization of the $700-3000 \mathrm{MHz}$ frequency band with different utilization percentage at each location. Furthermore, the measurements confirm that the spectrum occupancy highly depends on the time, frequency, and location. As a result, multidimensional spectrum measurement and analysis are vital for accurate spectrum utilization modeling and performance evaluation of CR systems.
\end{abstract}

\section{INTRODUCTION}

The continued success of wireless industry is placing more demands on an already scarse and valuable resource, viz., the Radio Frequecy (RF) spectrum. One of the most promising technologies for efficient spectrum utilization is Cognitive Radio (CR) [1] of which, spectrum awareness is one of the most prominent features [2]. Modeling of spectrum occupancy over multiple dimensions such as time, frequency, and space is crucial for the accurate development and performance evaluation of CR systems. Although it is possible to infer some information from spectrum licenses and regulations, it is not possible to estimate the occupation of spectrum accurately (even for licensed bands) because of the dynamic nature of its utilization. Therefore, it is essential to monitor the spectrum and measure the occupancy, which not only aids further quantifying the potential benefits of CR systems but also develop models that assist in the development of such systems.

Some spectrum occupancy measurement campaigns have been reported in the literature [3]-[11]. The majority of these studies can be categorized as single band monitoring, time - frequency analysis, and indoor vs. outdoor measurements. For instance, in [5] and [6], the spectrum occupancy over

This study is supported by Qatar Telecom (Qtel). time and frequency is studied while [4] studies the spectrum occupancy for indoor vs. outdoor considering time and frequency domains. Additionally, Industrial, Scientific and Medical (ISM, from $2400 \mathrm{MHz}$ to $2483 \mathrm{MHz}$ frequency range) band is one of the bands that its occupancy had been measured extensively in the literature such as in [12]. What is missing though, is the study of the spectrum occupancy over the spatial domain concurrently with the variable nature of RF spectrum occupancy over time, and frequency.

In this paper, a wideband multi-dimensional spectrum measurement campaign is performed to study the variations of RF spectrum occupancy over spatial, time, and frequency domains simultaneously. We report the results from the measurement campaign conducted for three consecutive days considering the spectrum between $700-3000 \mathrm{MHz}$ at four different locations in the State of Qatar. The results are presented in the form of power spectral density (PSD) for the given entire measured spectrum, bandwidth utilization over time, and 3D plot of spectral power variations over frequency and time for each location.

The remainder of the paper is organized as follows; in Section II, measurement setup and procedure are described along with the geographical characterization of the measurements. This is followed by presenting and discussing the measurement results in Section III. Finally, the conclusions are presented in Section IV.

\section{Measurement Setup and Environments}

\section{A. Measurement Setup}

The measurement setup and settings used are identical for each location and the measurement setup at one of the locations is shown in Fig. 1. In the setup, a Rohde \& Schwarz FSH6 Portable Spectrum Analyzer (SA) is connected to a laptop via a USB-optical cable. The SA is also connected to a very high performance discone antenna (AOR DA5000) with a $700-3000 \mathrm{MHz}$ frequency range and omni-directional horizontal receive capability. Table I shows the settings of the $\mathrm{SA}$, which is configured to measure the received signal power and store into laptop in real-time. 
TABLE I

SPECTRUM ANALYZER SETTINGS

\begin{tabular}{|c|c|}
\hline Center Frequency & $1.85 \mathrm{GHz}$ \\
\hline Frequency Span & $2.3 \mathrm{GHz}$ \\
\hline Resolution Bandwidth & $300 \mathrm{kHz}$ \\
\hline Video Bandwidth & $300 \mathrm{kHz}$ \\
\hline Sweep Time & $128 \mathrm{~ms}$ \\
\hline
\end{tabular}

$128 \mathrm{msec}$ of sweep time is selected as an optimum period considering the rise and fall times of sweeping band-pass filter and frequency resolution requirements. However, the rate of data collection is set to 1 reading per minute, where the entire spectrum of the $700-3000 \mathrm{MHz}$ band is swept at once with this sweep-and-dump rate. This was done for three consecutive days spanning just before and after the weekend ${ }^{1}$ which covers high as well as low population activity that can be reflected in spectrum utilization. Note that the selected frequency range is an active part of the spectrum where most of the current wireless communications systems are deployed.

Several software packages are used throughout the study. The Rohde \&S chwarz FSHview is used to save the measurement data obtained by the SA in the laptop. Furthermore, MATLAB software package is used for data post-processing and analysis.

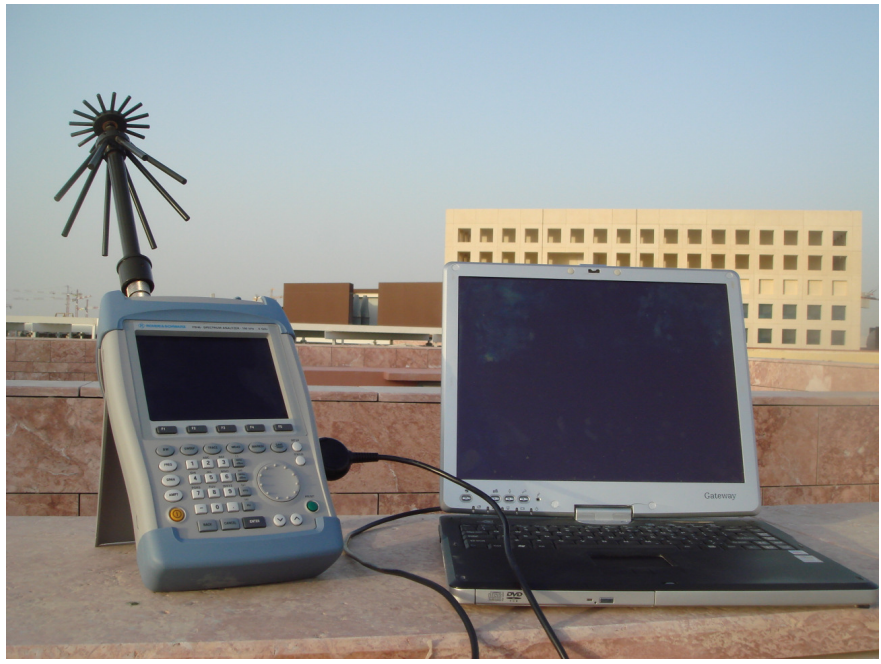

Fig. 1. Measurement setup at Location 1.

\section{B. Measurement Procedure and Geographical Locations}

Data was collected concurrently for the entire spectrum in four different geographical locations 6:00 PM local Qatar time $(\mathrm{GMT}+3)$ March 19, 2009 and 6:00PM March 22, 2009. This was done in order to study and characterize the variable nature

\footnotetext{
${ }^{1}$ The official weekend days in the State of Qatar are Friday and Saturday. In addition, the afternoon term is loosely used in this paper and it is mainly for 1:00PM-4:00PM time slot. Note that this time slot is a common but unofficial mid-day break in the State of Qatar due to extreme weather conditions such as high temperature and humidity.
}

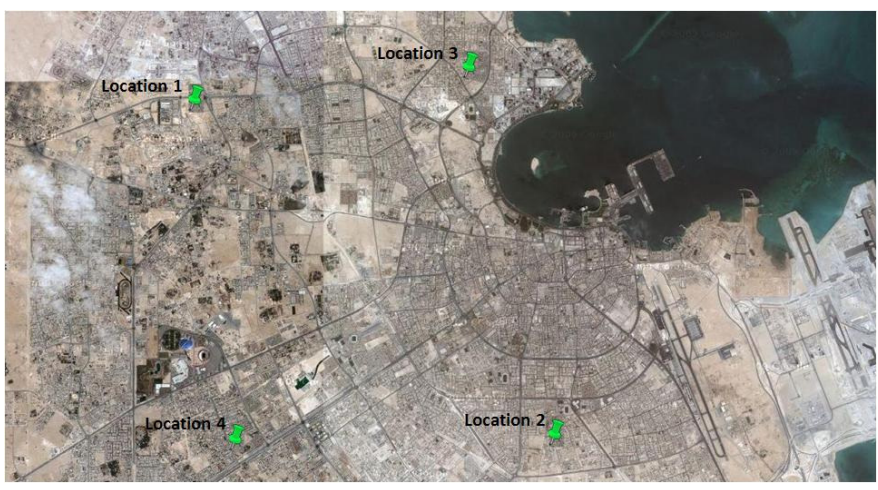

Fig. 2. Aerial map showing the measurement locations (Courtesy of Google Inc.).

of RF spectrum occupancy over time, frequency, and space. The four locations are designated Location 1 to 4 and are selected in such a way to represent different environments in the Doha, the capital city of Qatar. An aerial map of Doha City that shows these four locations is given in Fig. 2. Location 1 is located in the west of Doha, and comprises a number of education campuses surrounded by vast open and generally flat spaces with some construction work going on in the campus and its immediate surroundings. There are no commercial/residential buildings or areas in the vicinity. In this area, the measurement data is collected on the roof of Texas A\&M University at Qatar (TAMUQ) Engineering building. Location 2 is in the south east of Doha and is a busy commercial area. Different radar (aviation) and communications systems are deployed and as such it is an electromagnetically rich environment. Location 3 is closer to what is considered the downtown of Doha and consists of high towers (average 30 floors + ) and large commercial centers in addition to a hospital and a police station. Location 4 is in the south of Doha and consist of factories and workshops. For each location, 4320 different readings of the $700-3000 \mathrm{MHz}$ spectrum are collected during the measurement campaign.

The clock of each measurement setup is synchronized prior to placing them in their respective measurement locations. In addition, the measurement at all locations is started and stopped synchronously.

\section{Measurement Results and Discussion}

The results are presented with the help of Figures 3 to 8. Figure 3 shows the results of the average (PSD) over the entire measured spectrum for all locations. Figure 4 shows the utilization of the bandwidth over the three-day measurement period, again, for all locations. The remaining Figures 5 to 8 show PSD as a function of time and frequency for Location 1 to 4 , respectively.

The average PSD of Figure 3 is obtained by averaging all the readings that are collected during the three-day measurement period for each of the locations and the results are superimposed. The utilized (occupied) bandwidth percentage in $700-3000 \mathrm{MHz}$ spectrum over the three-day measurement 


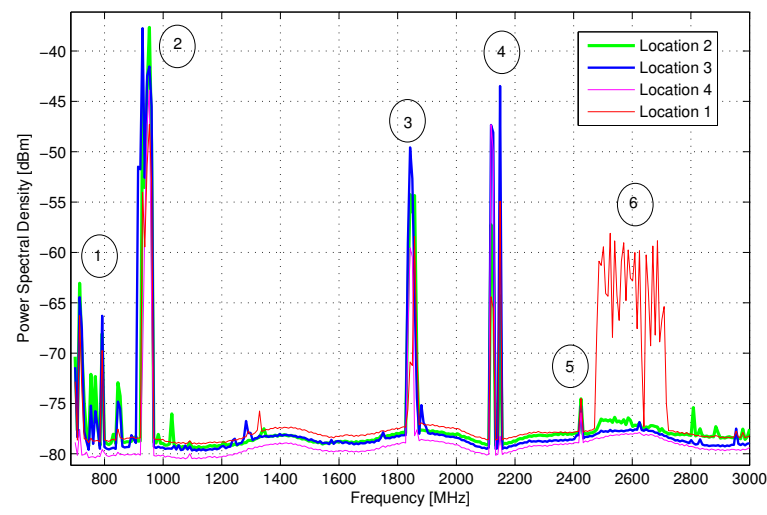

Fig. 3. Average power spectral density vs frequency at four locations from March 19, 2009 6.00PM to March 22, 2009 6.00PM (Markers: 1. TV broadcast, 2. GSM, 3. GSM 1800, 4. 3G, 5. Wi-Fi, 6. WiMAX bands).

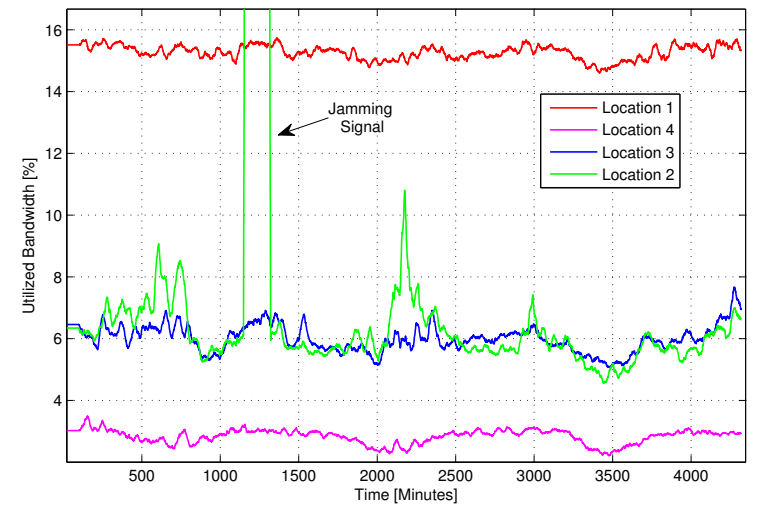

Fig. 4. Utilized bandwidth vs time at four locations from March 19, 2009 6.00PM to March 22, 2009 6.00PM.

period is calculated for each spectrum reading as follows; first, a threshold level is set in order to determine the utilized bandwidth. We measure the thermal noise of the measurement system, which is found to be $-78 \mathrm{dBm}$ over $700-3000 \mathrm{MHz}$ spectrum. However, a margin of $3 \mathrm{~dB}$ is considered for determining the final threshold value in order to account any unforeseen effects and variations [4]. As a result, $-75 \mathrm{dBm}$ is used as threshold throughout this study. If the measured PSD in a certain frequency is above this threshold, then this band is reported as utilized band otherwise it is considered as idle. In the following sections, the measurement results for each location are presented and discussed.

\section{A. Location 1}

The average PSD over the frequency spectrum for this location is shown in Fig. 3. The TV broadcast channels are observed in the band around $800 \mathrm{MHz}$. In addition, strong downlink (base station to mobile station) GSM channels are clearly identified in $950 \mathrm{MHz}$ and $1800 \mathrm{MHz}$ bands. In the bands around $2120 \mathrm{MHz}, 3 \mathrm{G} / \mathrm{UMTS}$ downlink channels are observed. Moreover, Wi-Fi channels with weak signal strength are observed at $2400 \mathrm{MHz}$ band. Finally, WiMAX channels are clearly identified in $2470-2720 \mathrm{MHz}$ band. These are clearly marked in the figures for ease of reference.

The utilized bandwidth over three consecutive days for this location is shown in Fig. 4. The highest percentage of the utilized bandwidth that is observed is $15.7 \%$ and the lowest percentage of the utilized bandwidth that is observed is 14.6 $\%$ suggesting little variation in the utilization of the bandwidth over the measurement period.

The 3D plot of PSD over the three days and the frequency spectrum is shown in Fig. 5. When the focus is on 3G/UMTS signals, it can be indicated that these signals are strong for the first 700 minutes (approximately 12 hours) and then their PSD reduces to $-65 \mathrm{dBm}$ level for the rest of the measurement time. This may be due to the high demand of normal services in addition to mobile broadband (using HSPA, the High Speed Packet Access) and are aligned with the usual traffic trends of one of the mobile network operators.

\section{B. Location 2}

The average PSD over the frequency spectrum for this location is given in Fig. 3 and it excludes a jamming signal that will be discussed later. Similar results to Location 1 are obtained except for the lack of WiMAX channels. In addition, strong TV broadcasting signals are identified in this location.

The utilized bandwidth over the three-day measurement period is shown in Fig. 4. Notice that a jamming signal that started around 1:30 PM and stopped around 4.30PM on March 20, 2009 was observed ${ }^{2}$. This jamming signal occupied $70 \%$ of the bandwidth and it was strong in the frequency range below $1 \mathrm{GHz}$. On the other hand, the peak time for this location is found to be in the afternoon and the utilized bandwidth varies around $7 \%$, which is about half that in Location 1. Moreover, the transmission on the TV broadcasting band is strong in this region. Finally, Fig. 6 shows the 3D plot of PSD over the time and frequency in this location. Note that the jamming signal is also clearly identified in this figure.

\section{Location 3}

The average PSD over the frequency spectrum for this location is shown in Fig. 3. The results in this location are similar to these in the Location 2 apart from the absence of any jamming signal. In Fig. 4, the utilized bandwidth over the three days is shown. According to the results, the utilized bandwidth hovers around $6 \%$ and low activity in the early mornings is observed in this location. The peak time is again in the afternoon and the highest percentage of utilized bandwidth is observed on Sunday afternoon (March 22, 2009). Note that the utilized bandwidth reaches up to $7 \%$ at peak time in this location. Finally, Fig. 7 shows the 3D PSD plot as a function of time and frequency for this location.

\footnotetext{
${ }^{2}$ Determining the source and location of this jamming signal is out of scope of this study.
} 


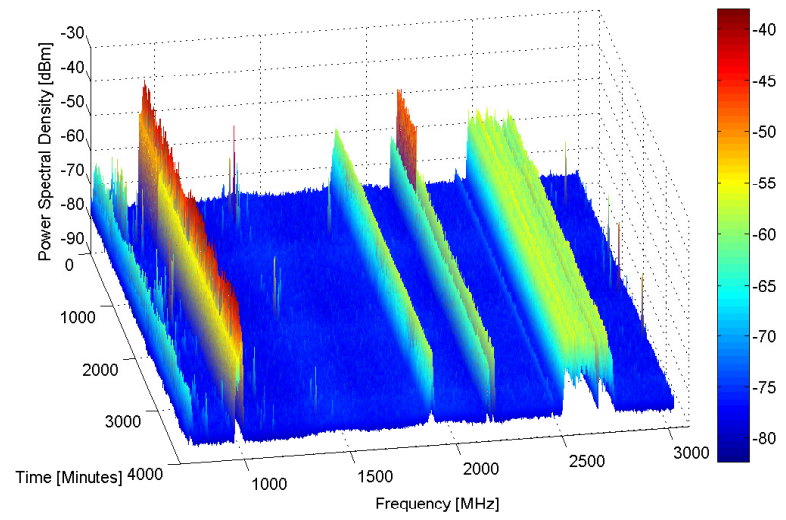

Fig. 5. Power spectral density vs time vs frequency at location 1 from March 19, 2009 6.00PM to March 22, 2009 6.00PM.

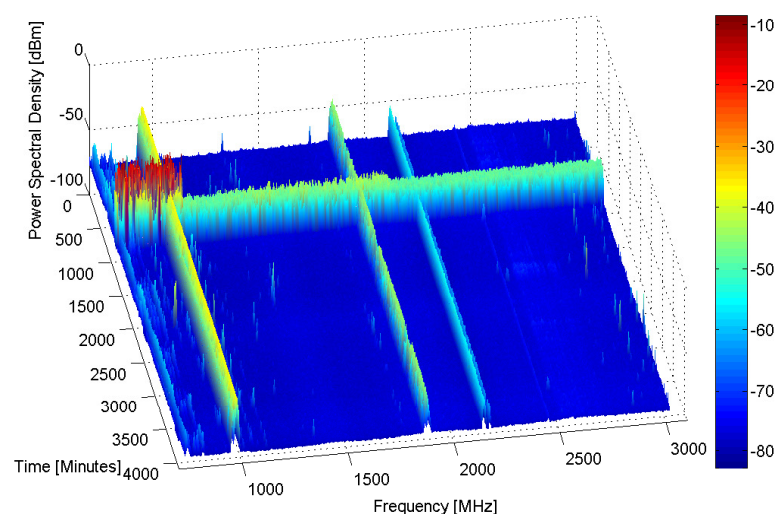

Fig. 6. Power spectral density vs time vs frequency at location 2 from March 19, 2009 6.00PM to March 22, 2009 6.00PM.

\section{Location 4}

The average PSD as a function of frequency, the utilized bandwidth over the three days, and 3D plot of PSD over time and frequency are shown in Figs. 3, 4, and 8, respectively. According to the results in Fig. 3, downlink GSM channels are clearly observed in $950 \mathrm{MHz}$ and $1800 \mathrm{MHz}$ bands for this region. Furthermore, downlink 3G/UMTS channels are identified in the band around $2100 \mathrm{MHz}$. Note that weak TV broadcasting and Wi-Fi signals are obtained in this location. It can be concluded that the majority of the spectrum is occupied by the Mobile communications systems in this location. In addition, the utilized bandwidth in this region varies around $3 \%$ with the peak at $3.5 \%$. Finally, the utilized bandwidth curve shows the visible cycles at day and night.

The results show that the average PSD of GSM and 3G/UMTS frequency bands are similar at four locations with varying gains due to distance difference between each location and the closest base stations. More interestingly though is the absence of any measurements of uplink 3G/UMTS and GSM signals. The 3G/UMTS uplink is $190 \mathrm{MHz}$ below the transmit

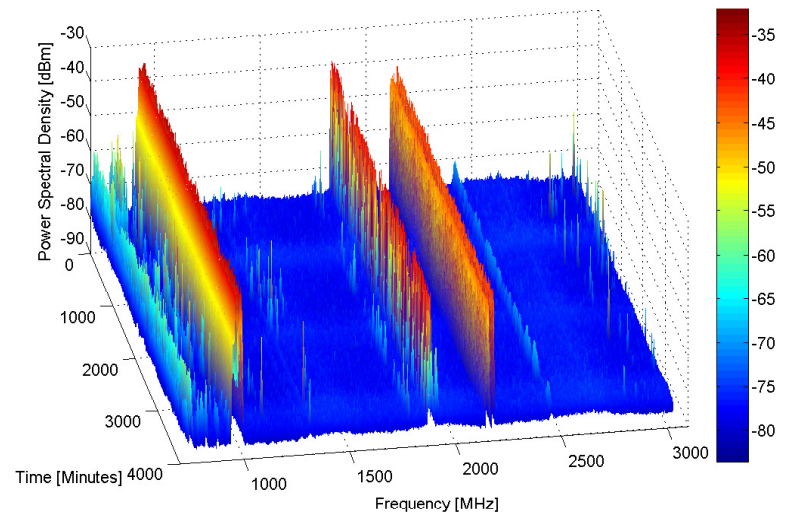

Fig. 7. Power spectral density vs time vs frequency at location 3 from March 19, 2009 6.00PM to March 22, 2009 6.00PM.

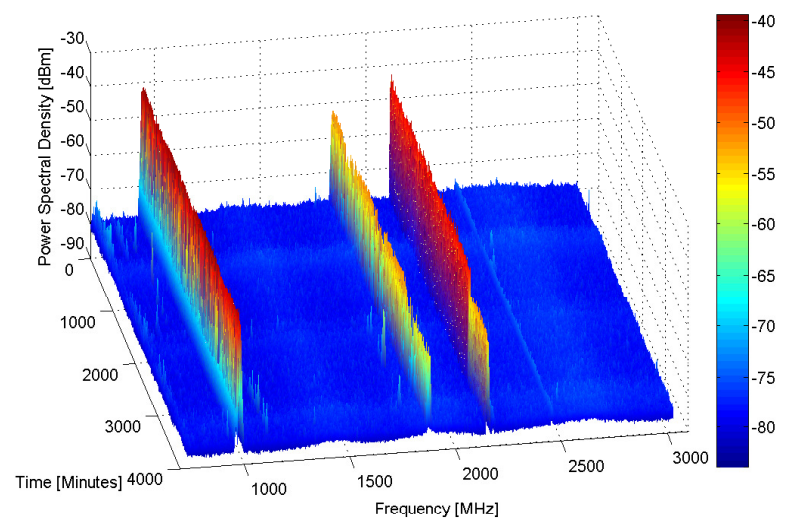

Fig. 8. Power spectral density vs time vs frequency at location 4 from March 19, 2009 6.00PM to March 22, 2009 6.00PM.

band and the GSM1800 has a $75 \mathrm{MHz}$ duplex distance ([13], see specifications such as 25.101, 25.104, 45.002, 45.005, 45.008). The GSM 900 has a $45 \mathrm{MHz}$ separation. The reason for lack of measurements can be manifold. For start the TDMA nature of GSM means that the uplink is transmitting a very short burst every $4.615 \mathrm{~ms}$ but this transmission rate is further reduced when Discontinuous Transmission (DTX) is used and Slow Frequency Hopping (SFH) further compounds the detection problem. Additionally, the uplink power control dynamic range is much larger than that of the downlink meaning that the mobile can use far less power than the base station. All of this is further compounded by the high relative noise floor of the receiver system but this is mainly due to the high noise figure of the SA. As for 3G/UMTS the large processing gain allows the mobile to transmit at very low powers and once again the noise floor of the system is a factor.

The authors are of the opinion that further characterization of Frequency Division Duplex systems can help in CR systems, where the uplink bands can be utilized for, e.g. low power Time Division Duplex systems (TDD). Algorithms can 
be derived to make decisions based on the characteristics of the downlink spectrum as well as the sensing of the utilization in the uplink band.

The average PSD for low frequency bands around $700 \mathrm{MHz}$ are similar for all the locations except Location 4 . The average PSD in this band is low for the Location 4 due to the relatively large distance to the TV stations. In addition, average PSD for Wi-Fi frequency band is similar for all the four locations and this is justified by the existence of Wi-Fi services at the four locations. Finally, strong WiMAX signals are only detected in Location 1.

According to the results in Fig. 4, Location 1 registers the highest bandwidth utilization in the $700-3000 \mathrm{MHz}$ band. On the other hand, Location 2 and 3 show similar daily behavior between them due to their similar environments such as being close to sea, commercial centers, and having major traffic roads. 02:00PM - 06:00PM time period show peaks at all four locations while the minimum activity is observed just before the sunrise. As for Location 4, it registers the lowest bandwidth utilization percentage among all the locations. Most of the electromagnetic activity in this region is originated from only mobile phone traffic. In Location 2, there is an unexpected increase in the utilized bandwidth at the afternoon of March 20, 2009 (Friday) because of jamming signal, which occupies $70 \%$ of the $700-3000 \mathrm{MHz}$ spectrum.

One note is that the authors understand that the percentage occupancy of the spectrum is highly dependent upon the choice of extent and the absolute location of the measured bandwidth but nonetheless, gives a useful metric.

\section{CONCLUSiOns}

A comprehensive wideband spectrum occupancy measurement is performed over multiple dimension (time, frequency and space) concurrently. The results show that the spatial variation is significant in terms of utilized bandwidth in the $700-3000 \mathrm{MHz}$ spectrum over a three day measurement period. One location had the busiest spectrum occupancy, especially in the afternoons. Across four different locations, the bandwidth utilization varies between $4 \%$ and $15 \%$. Additionally, it is observed that bandwidth utilization is time variant with peak times ranging from afternoon to sunset in almost all of the locations. Furthermore, we observe an interesting event, which is a jamming signal in Location 2. Although the methodology can be refined in order to detect lower power signals and signals of a more transient nature, the measurement results show that the spectrum occupancy varies according to time, frequency, and space. Hence, multidimensional spectrum occupancy measurement and analysis must be seen as critical and of high importance for the accurate modeling of spectrum utilization, and development and performance evaluation of CR systems. Finally, the current study will be extended to model spectrum utilization and prediction of unused bands considering spatial domain.

\section{ACKNOWLEDGMENTS}

The authors would like to thank Mr. Laith Abuhantash, Mr. Mohammad Al-Mulla, Mr. Omar Al-Mulla, Mr. Abdulrahman Jolo, and Mr. Ali Ahmed for collecting data. In addition, we wish to thank Qatar Foundation, Qatar Ministry of Interior, and ictQatar for providing us the required legal authorizations to conduct the measurement campaign.

\section{REFERENCES}

[1] J. Mitola and G. Q. Maguire, "Cognitive radio: Making software radios more personal," IEEE Personal Commun. Mag., vol. 6, no. 4, pp. 13-18, August 1999.

[2] Z. Quan, S. Cui, H. V. Poor, and A. H. Sayed, "Collaborative wideband sensing for cognitive radios," IEEE Signal Processing Magazine, vol. 25 , no. 6, pp. 60-73, 2008.

[3] M. Islam, G. L. Tan, F. Chin, B. E. Toh, Y.-C. Liang, C. Wang, Y. Y. Lai, X. Qing, S. W. Oh, C. L. Koh, and W. Toh, "Spectrum survey in singapore: Occupancy measurements and analyses," in Proc. of International Conference on Cognitive Radio Oriented Wireless Networks and Communications (CROWNCOM), Singapore, May 2008.

[4] M. Wellens, J. Wu, and P. Mahonen, "Evaluation of spectrum occupancy in indoor and outdoor scenario in the context of cognitive radio," in Proc. of International Conference on Cognitive Radio Oriented Wireless Networks and Communications (CROWNCOM), Orlando, FL, USA Aug. 2007.

[5] T. Erpek, M. Lofquist, and K. Patton, "Spectrum occupancy measurements: Loring commerce centre, limestone, maine, sep. 18-20, 2007," Shared Spectrum Company, Tech. Rep., 2007.

[6] M. A. McHenry, P. A. Tenhula, D. McCloskey, D. A. Roberson, and C. S. Hood, "Chicago spectrum occupancy measurements \& analysis and a long-term studies proposal," in Proc. of Workshop on Technology and Policy for Accessing Spectrum (TAPAS), Boston, USA, Aug. 2006.

[7] R. I. C. Chiang, G. B. Rowe, and K. W. Sowerby, "A quantitative analysis of spectral occupancy measurements for cognitive radio," in Proc. IEEE Vehicular Technology Conference, Dublin, Ireland, Apr. 2007.

[8] S. W. Ellingson, "Spectral occupancy at vhf: Implications for frequencyagile cognitive radios," in Proc. of IEEE Vehicular Technology Conference (VTC), Dallas, USA, Sept. 2005, pp. 1379-1382.

[9] A. Petrin and P. G. Steffes, "Analysis and comparison of spectrum measurements performed in urban and rural areas to determine the total amount of spectrum usages," in Proc. of International Symposium on Advanced Radio Technologies, Boulder, USA, March 2005, pp. 9-12.

[10] J. Do, D. M. Akos, and P. K. Enge, "L and s bands spectrum survey in the san francisco bay area," in Proc. of IEEE Position, Location and Navigation Symposium, Apr. 2004.

[11] F. H. Sanders, "Broadband spectrum surveys in denver, co, san diego, ca, and los angeles, ca: Methodology, analysis, and comparative results,' in Proc. of IEEE Symposium on Electromagnetic Compatibility, 1998.

[12] F. Chinchilla, M. Lindsey, and M. Papadopouli, "Analysis of wireless information locality and association patterns in a campus," in In Proceedings of the Conference on Computer Communications (IEEE Infocom), Hong Kong, March 2004.

[13] “3rd generation partnership project (3gpp)," 2009. [Online]. Available: http://www.3gpp.org/ 